\title{
Global existence of 2D nonhomogeneous incompressible magnetohydrodynamics with vacuum
}

\author{
Menglong Su ${ }^{1 *}$, Xiaohui Qian ${ }^{2}$ and Jian Wang ${ }^{3}$
}

\section{"Correspondence:}

mlsulynu@163.com

${ }^{1}$ School of Mathematical Sciences,

Luoyang Normal University,

Luoyang, 471022, P.R. China

Full list of author information is

available at the end of the article

\begin{abstract}
In this paper, we prove the existence of global strong solutions to the Cauchy problem of 2D incompressible magnetohydrodynamics (MHD) flows. Here, we emphasize that the initial density $\rho_{0}$ is permitted to contain vacuum states, and the initial velocity $u_{0}$ and magnetic fields $H_{0}$ can be arbitrarily large.
\end{abstract}

Keywords: global strong solutions; 2D incompressible magnetohydrodynamics flows; vacuum states

\section{Introduction}

The mathematical model of magnetohydrodynamics (MHD) is used to simulate the motion of a conducting fluid under the effect of the electromagnetic field and has a very wide range of applications in astrophysics, plasma, and so on. The governing equations of nonhomogeneous MHD can be stated as follows [1,2]:

$$
\begin{aligned}
& \rho_{t}+\operatorname{div}(\rho u)=0, \\
& (\rho u)_{t}+\operatorname{div}(\rho u \otimes u)-\mu \Delta u+\nabla P=(B \cdot \nabla) B, \\
& B_{t}+(u \cdot \nabla B)-(B \cdot \nabla) u=v \Delta B, \\
& \operatorname{div} u=0, \quad \operatorname{div} B=0
\end{aligned}
$$

with $t \geq 0$ and $x=\left(x_{1}, x_{2}\right) \in \mathbb{R}^{2}$. The unknown functions $\rho, u, P$, and $B$ denote the fluid density, velocity, pressure, and magnetic field, respectively. The constant $\mu>0$ is the viscosity coefficient. The constant $v>0$ is the resistivity coefficient, which is inversely proportional to the electrical conductivity constant and acts as the magnetic diffusivity of magnetic fields. Without loss of generality, we set $\mu=v=1$ throughout the paper. In this paper, we assume the state equation $P=P(\rho)=a \rho^{\gamma}(a>0, \gamma>1)$ and study the Cauchy problem. Without loss of generality, we assume that $a=1$. In this paper, we consider the Cauchy problem for (1)-(4) with $(\rho, u, B)$ with given initial data $\rho_{0}, B_{0}$, and $u_{0}$, as

$$
\rho(x, 0)=\rho_{0}(x), \quad \rho u(x, 0)=\rho_{0} u_{0}(x), \quad B(x, 0)=B_{0}(x), \quad x \in \mathbb{R}^{2}
$$


and far-field behavior

$$
(\rho, u, B)(x, t) \rightarrow(\tilde{\rho}, 0,0) \quad \text { (in some weak sense), as }|x| \rightarrow \infty,
$$

where $\tilde{\rho}$ is some fixed positive constant.

The global well-posedness and dynamical behaviors of MHD system are rather difficult to investigate because of the strong coupling and interplay interaction between the fluid motion and the magnetic fields. Recently, there is much more important progress on the mathematical analysis of these topics for the (nonhomogeneous or homogeneous) MHD system (see, for example, [3-20]). Here, we only mention some of them. Kawashima [14] obtained the global existence of smooth solutions in the two-dimensional case when the initial data are a small perturbation of some given constant state. Li-Xu-Zhang showed in [15] the global well-posedness and large-time behavior of classical solutions to the Cauchy problem of compressible MHD for regular initial data with small energy but possibly large oscillations. In [9, 18], Hoff and Tsyganov obtained the global existence and uniqueness of weak solutions with small initial energy. Umeda-Kawashima-Shizuta [17] studied the global existence and time decay rate of smooth solutions to the linearized two-dimensional compressible MHD equations. The optimal decay estimates of classical solutions to the compressible MHD system were obtained by Zhang-Zhao [20] when the initial data are close to a nonvacuum equilibrium. Hu-Wang $[10,11]$ and Fan-Yu [8] proved the global existence of renormalized solutions to the compressible MHD equations for general large initial data. When the viscosity and resistivity go to zero, Zhang [19] showed that the solution of the Cauchy problem for the nonhomogeneous incompressible MHD system converges to the solution of the ideal MHD system and the convergence rate was also obtained. Craig-Huang-Wang [7] obtained the global existence and uniqueness of strong solutions for initial data with small $\dot{H}^{\frac{1}{2}}$-norm in the bounded or unbounded domain in $\mathbb{R}^{3}$.

In [12], Huang-Wang considered the global strong solutions to (1)-(4) in the bounded domain with suitable boundary conditions on $u$ and $B$. Their arguments actually depend on the size of the domain, and so they cannot be applied to the Cauchy problem directly. Then one natural question may be raised: whether the global strong solutions exist in the whole space $\mathbb{R}^{2}$. Here, we want to answer the question. Our main result is stated as follows.

Theorem 1.1 Assume that the initial data $\rho_{0}, u_{0}$, and $B_{0}$ satisfy

$$
\left\{\begin{array}{l}
\rho_{0} \geq 0, \quad\left(\rho_{0}-\tilde{\rho}, u_{0}, B_{0}\right) \in H^{2}\left(\mathbb{R}^{2}\right) \\
\operatorname{div} u_{0}=\operatorname{div} B_{0}=0 \\
-\Delta u_{0}+\nabla P_{0}-B_{0} \cdot \nabla B_{0}=\rho_{0}^{\frac{1}{2}} g
\end{array}\right.
$$

where $\left(\nabla P_{0}, g\right) \in L^{2}\left(\mathbb{R}^{2}\right)$. Then for any given $0<T<\infty$, there exists a unique global strong solution $(\rho, u, P, B)$ of (1)-(6) such that

$$
\left\{\begin{array}{l}
\rho \in C\left([0, T] ; H^{2}\left(\mathbb{R}^{2}\right)\right), \quad(u, B) \in C\left([0, T] ; H^{2}\left(\mathbb{R}^{2}\right)\right), \\
P \in C\left([0, T] ; H^{1}\left(\mathbb{R}^{2}\right)\right) \cap L^{2}\left(0, T ; H^{2}\left(\mathbb{R}^{2}\right)\right), \quad\left(u_{t}, B_{t}\right) \in L^{2}\left(0, T ; H^{1}\left(\mathbb{R}^{2}\right)\right), \\
\left(\rho_{t}, \sqrt{\rho} u_{t}, B_{t}\right) \in L^{\infty}\left(0, T ; L^{2}\left(\mathbb{R}^{2}\right)\right) .
\end{array}\right.
$$


The proof of Theorem 1.1 is mainly based on a critical Sobolev inequality of logarithmic type which was recently proved by Huang-Wang [12] and is originally due to BrezisWainger [21]. The main difficulty compared with [12] is that we should bound all the desired estimates without the restriction on the size of the domain, especially that the Poincaré inequality is not the same from the bounded domain to the whole spaces.

For convenience, we explain the notions used throughout this paper. Set

$$
\int f d x \triangleq \int_{\mathbb{R}^{2}} f d x
$$

The standard homogeneous and inhomogeneous Sobolev spaces are defined as follows:

$$
\left\{\begin{array}{l}
L^{r}=L^{r}\left(\mathbb{R}^{2}\right), \quad D^{k, r}\left(\mathbb{R}^{2}\right)=\left\{v \in L_{\mathrm{loc}}^{1}\left(\mathbb{R}^{2}\right) \mid \nabla^{k} v \in L^{r}\left(\mathbb{R}^{2}\right)\right\}, \\
D^{1}=D^{1,2}, \quad W^{k, r}=W^{k, r}\left(\mathbb{R}^{2}\right), \quad H^{k}=W^{k, 2},
\end{array}\right.
$$

for $1 \leq r \leq \infty$ and $k \geq 1$.

The paper is organized as follows. In Section 2, we state some well-known inequalities and basic facts which will be used frequently later. The proof of Theorem 1.1 will be cast in Section 3.

\section{Preliminaries}

In this section, we list some useful lemmas which will be frequently used in the next sections. We start from the local existence of strong solutions, which is similar to [4] or [14].

Lemma 2.1 Assume that the conditions of Theorem 1.1 hold. Then there exists a positive time $T_{0}$ such that the Cauchy problem (1)-(6) admits a unique strong solution on $\mathbb{R}^{2} \times$ $\left[0, T_{0}\right]$.

Next is the well-known Gagliardo-Nirenberg inequality (see [22]).

Lemma 2.2 For $f \in H^{1}\left(\mathbb{R}^{2}\right)$, we have for any $2 \leq p<\infty$

$$
\|f\|_{L^{p}}^{p} \leq C(p)\|f\|_{L^{2}}^{2}\|\nabla f\|_{L^{2}}^{p-2}
$$

where $C(p)$ is a positive constant depending only on $p$. In addition, iff $\in W^{1, p}\left(\mathbb{R}^{2}\right) \cap H^{2}\left(\mathbb{R}^{2}\right)$ with $p>2$, then there exists a universal positive constant $C$ such that

$$
\|f\|_{L^{\infty}} \leq C\|f\|_{W^{1, p}\left(\mathbb{R}^{2}\right)} \leq C\|f\|_{H^{2}\left(\mathbb{R}^{2}\right)} .
$$

Next, we list the Poincaré type inequality, which yields $\|v\|_{L^{2}\left(\mathbb{R}^{2}\right)}$ even when the vacuum states appear.

Lemma 2.3 Assume that $\rho-\tilde{\rho} \in L^{2}\left(\mathbb{R}^{2}\right) \cap L^{\infty}\left(\mathbb{R}^{2}\right)$ with $\rho(x) \geq 0, \nabla v \in L^{2}\left(\mathbb{R}^{2}\right)$ and $\sqrt{\rho} v \in$ $L^{2}\left(\mathbb{R}^{2}\right)$. Then

$$
\|v\|_{L^{2}} \leq C\left(\|\sqrt{\rho} v\|_{L^{2}}+\|\nabla v\|_{L^{2}}\right)
$$

where $C$ depends only on $\tilde{\rho},\|\rho-\tilde{\rho}\|_{L^{2}}$, and $\|\rho-\tilde{\rho}\|_{L^{\infty}}$. 
Proof The proof of this lemma can easily be deduced by (9), Hölder's inequality and the following equality:

$$
\tilde{\rho} \int|v|^{2} d x=\int \rho|v|^{2} d x-\int(\rho-\tilde{\rho})|v|^{2} d x,
$$

so the details are omitted here.

In the following, in order to improve the regularity of the velocity, we need to use the estimates of the Stokes equations. We refer the reader to [23, 24] for details.

Lemma 2.4 Consider the following stationary Stokes equations:

$$
-\Delta U+\nabla P=f, \quad \operatorname{div} U=0, \quad \text { in } \mathbb{R}^{2} .
$$

Then for any $f \in W^{m, p}(p>1)$, there exists a positive constant $C$, depending only on $m$ and $p$, such that

$$
\left\|\nabla^{2} U\right\|_{W^{m, p}}+\|\nabla P\|_{W^{m, p}} \leq C\|f\|_{W^{m, p}} .
$$

To improve the regularity of the magnetic fields, we need the following result on the elliptic system.

Lemma 2.5 Assume that $B \in H^{1}$ is a weak solution of the Poisson equations

$$
\Delta B=g, \quad \text { in } \mathbb{R}^{2},
$$

where $g \in L^{q}(1<q<\infty)$. Then we have

$$
\|B\|_{W^{2, q}} \leq C\|g\|_{L^{q}},
$$

with some constant $C$ depending only on $q$.

To bound the $L^{2}$-norm of the gradient of the velocity, we will apply a critical Sobolev inequality of logarithmic type which was proved by Huang-Wang [12]. This is the key tool for the proof of Theorem 1.1.

Lemma 2.6 For $q>2$ and $0 \leq s<t<\infty$, assume that $f \in L^{2}\left(s, t ; H^{1}\right) \cap L^{2}\left(s, t ; W^{1, q}\right)$. Then there exists a positive constant $C(q)$, independent of $s$ and $t$, such that

$$
\|f\|_{L^{2}\left(s, t ; L^{\infty}\right)} \leq C\left(1+\|f\|_{L^{2}\left(s, t ; H^{1}\right)}\left(\ln ^{+}\|f\|_{L^{2}\left(s, t ; W^{1, q}\right)}\right)^{\frac{1}{2}}\right) .
$$

\section{Proof of Theorem 1.1}

This section is devoted to obtaining the proof of Theorem 1.1. According to Lemma 2.1, a local strong solution of the Cauchy problem (1)-(6) exists. Suppose $T^{*}$ is the first blowup time of the strong solution $(\rho, u, P, B)$ to the Cauchy problem, it suffices to prove there 
actually exists a generic positive constant $M(0<M<\infty)$, depending only on the initial data $\left(\rho_{0}, u_{0}, B_{0}\right)$ and $T^{*}$, such that

$$
\begin{aligned}
\Phi \triangleq & \sup _{0 \leq t \leq T}\left(\|\rho-\tilde{\rho}\|_{H^{2}}^{2}+\|(u, B)\|_{H^{2}}^{2}+\left\|\sqrt{\rho} u_{t}\right\|_{L^{2}}^{2}\right) \\
& +\int_{0}^{T}\left(\|(u, B)\|_{H^{3}}^{2}+\left\|u_{t}\right\|_{H^{1}}^{2}+\left\|H_{t}\right\|_{H^{1}}^{2}\right) d t \leq M,
\end{aligned}
$$

where $0<T<T^{*}$. Then due to the local existence theorem (Lemma 2.1), it can easily be shown that the strong solution can be extended beyond $T^{*}$. This conclusion contradicts the assumption on $T^{*}$. Thus, the strong solution exists globally on $\mathbb{R}^{2} \times[0, T]$ for any $0<T<\infty$. Hence the proof of Theorem 1.1 is therefore completed.

The proof of (15) is based on a series of lemmas. For simplicity, throughout the remainder of this paper, we denote by $C$ a generic constant which depends only on the initial data and $T^{*}$ and may change from line to line.

First, the $L^{\infty}$-norm of the density can be obtained easily by using the method of characteristics, we list the following lemma without proof.

Lemma 3.1 For every $0<T<T^{*}$, we have

$$
0 \leq \sup _{0 \leq t \leq T}\|\rho\|_{L^{\infty}} \leq\left\|\rho_{0}\right\|_{L^{\infty}}
$$

Next, the basic energy inequalities are used.

Lemma 3.2 For every $0<T<T^{*}$, we have

$$
\sup _{0 \leq t \leq T}\left(\|\sqrt{\rho} u\|_{L^{2}}^{2}+\|B\|_{L^{2}}\right)+\int_{0}^{T}\left(\|\nabla u\|_{L^{2}}^{2}+\|\nabla B\|_{L^{2}}^{2}\right) d t \leq C .
$$

The following estimates are the key estimates in the proof of Theorem 1.1, which depends on the critical Sobolev inequality of logarithmic type (see Lemma 2.6).

Lemma 3.3 For every $0<T<T^{*}$, we have

$$
\sup _{0 \leq t \leq T}\left(\|u\|_{H^{1}}^{2}+\|B\|_{H^{1}}^{2}\right)+\int_{0}^{T}\left(\|\sqrt{\rho} \dot{u}\|_{L^{2}}^{2}+\left\|B_{t}\right\|_{L^{2}}^{2}\right) d t \leq C,
$$

where $\dot{f}=f_{t}+u \cdot \nabla f$ is the material derivative of $f$.

Proof First, multiplying (2) by $u_{t}$ and integrating the resultant equation by parts over $\mathbb{R}^{2}$ on $x$, one deduces that

$$
\frac{1}{2} \frac{d}{d t}\|\nabla u\|_{L^{2}}^{2}+\left\|\rho^{\frac{1}{2}} \dot{u}\right\|_{L^{2}}^{2}=\int \rho \dot{u}(u \cdot \nabla) u d x+\int(B \cdot \nabla) B \cdot u_{t} d x .
$$

For the first term on the right-hand side of (19), using Young's inequality and (16), one shows that

$$
\int \rho \dot{u}(u \cdot \nabla) u \leq \frac{1}{2}\|\sqrt{\rho} \dot{u}\|_{L^{2}}^{2}+C\|u\|_{L^{\infty}}^{2}\|\nabla u\|_{L^{2}}^{2} .
$$


Next, the second term can be deduced as follows:

$$
\begin{aligned}
\int(B \cdot \nabla B) \cdot u_{t} & =\frac{d}{d t} \int(B \cdot \nabla) B \cdot u d x-\int\left(B_{t} \cdot \nabla\right) B \cdot u d x-\int(B \cdot \nabla) B_{t} \cdot u d x \\
& =-\frac{d}{d t} \int(B \cdot \nabla) u \cdot B d x+\int\left(B_{t} \cdot \nabla\right) u \cdot B d x-\int(B \cdot \nabla) u \cdot B_{t} \\
& \leq-\frac{d}{d t} \int(B \cdot \nabla) u \cdot B d x+\frac{1}{4}\left\|B_{t}\right\|_{L^{2}}^{2}+C\|B\|_{L^{\infty}}^{2}\|\nabla u\|_{L^{2}}^{2} .
\end{aligned}
$$

Then, substituting the above two estimates into (19), one obtains

$$
\begin{aligned}
& \frac{1}{2} \frac{d}{d t}\|\nabla u\|_{L^{2}}^{2}+\frac{d}{d t} \int(B \cdot \nabla) u \cdot B d x+\frac{1}{2}\|\sqrt{\rho} \dot{u}\|_{L^{2}}^{2} \\
& \quad \leq \frac{1}{4}\left\|B_{t}\right\|_{L^{2}}^{2}+C\left(\|u\|_{L^{\infty}}^{2}+\|B\|_{L^{\infty}}^{2}\right)\|\nabla u\|_{L^{2}}^{2} .
\end{aligned}
$$

Multiplying (3) by $B_{t}$ and integrating over $\mathbb{R}^{2}$ by parts, one deduces that

$$
\begin{aligned}
\left\|B_{t}\right\|_{L^{2}}^{2}+\frac{d}{d t}\|\nabla B\|_{L^{2}}^{2} & =-\int u \cdot \nabla B \cdot B_{t} d x+\int B \cdot \nabla u \cdot B_{t} d x \\
& \leq \frac{1}{4}\left\|B_{t}\right\|_{L^{2}}^{2}+C\left(\|u\|_{L^{\infty}}^{2}+\|B\|_{L^{\infty}}^{2}\right)\left(\|\nabla u\|_{L^{2}}^{2}+\|\nabla B\|_{L^{2}}^{2}\right) .
\end{aligned}
$$

The term $\frac{d}{d t} \int(B \cdot \nabla) u \cdot B d x$ on the left-hand side of (20) cannot be determined positive or negative, thus we have to control it by some appropriate positive terms. Note that it follows from Gagliardo-Nirenberg inequality that we may deduce

$$
\begin{aligned}
\left|\int(B \cdot \nabla) u \cdot B d x\right| & \leq\|B\|_{L^{4}}^{2}\|\nabla u\|_{L^{2}} \leq C\|B\|_{L^{2}}\|B\|_{H^{1}}\|\nabla u\|_{L^{2}} \\
& \leq \frac{1}{4}\|\nabla u\|_{L^{2}}^{2}+C_{1}\|B\|^{2}\left(\|B\|^{2}+\|\nabla B\|^{2}\right) .
\end{aligned}
$$

Then multiplying (21) by $\left(2 C_{1} C_{0}+1\right)$, adding it to (20), and integrating the resulting equation over $(s, t)$ on time, we finally deduce that

$$
\begin{aligned}
& \left(\|\nabla u\|_{L^{2}}^{2}+\|\nabla B\|_{L^{2}}^{2}\right)(t)+\int_{s}^{t}\left(\left\|\rho^{\frac{1}{2}} \dot{u}\right\|_{L^{2}}^{2}+\left\|B_{t}\right\|_{L^{2}}^{2}\right) d \tau \\
& \quad \leq C\left(\|\nabla u\|_{L^{2}}^{2}+\|\nabla B\|_{L^{2}}^{2}\right)(s) \exp \left\{C \int_{s}^{t}\left(\|u\|_{L^{\infty}}^{2}+\|B\|_{L^{\infty}}^{2}\right)\right\} d \tau .
\end{aligned}
$$

To proceed, we have to estimate $\|u\|_{L^{\infty}}$ and $\|B\|_{L^{\infty}}$. First, due to (11), we obtain

$$
\|u\|_{L^{2}}^{2} \leq C\left(1+\|\nabla u\|_{L^{2}}^{2}\right)
$$

For convenience, we denote

$$
\Psi(t)=e+\sup _{0 \leq \tau \leq t}\left(\|u(\tau)\|_{H^{1}}^{2}+\|B(\tau)\|_{H^{1}}^{2}\right)+\int_{0}^{t}\left(\|\sqrt{\rho} \dot{u}(\tau)\|_{L^{2}}^{2}+\left\|B_{t}(\tau)\right\|_{L^{2}}^{2}\right) d \tau .
$$


Then, combining (17), (22), and (23), we conclude that

$$
\Psi(t) \leq C \Psi(s) \exp \left\{C \int_{s}^{t}\left(\|u\|_{L^{\infty}}^{2}+\|B\|_{L^{\infty}}^{2}\right) d \tau\right\} .
$$

To proceed, we have to get the appropriate bound on $\|u\|_{L^{\infty}}$ and $\|B\|_{L^{\infty}}$. Thus, due to (13), we obtain

$$
\begin{aligned}
\left\|\nabla^{2} B\right\|_{L^{2}} & \leq C\left(\left\|B_{t}\right\|_{L^{2}}+\|u\|_{L^{\infty}}\|\nabla B\|_{L^{2}}+\|B\|_{L^{\infty}}\|\nabla u\|_{L^{2}}\right) \\
& \leq C\left(\left\|B_{t}\right\|_{L^{2}}+\|u\|_{L^{2}}^{\frac{1}{2}}\left\|\nabla^{2} u\right\|_{L^{2}}^{\frac{1}{2}}\|\nabla B\|_{L^{2}}+\|B\|_{L^{2}}^{\frac{1}{2}}\left\|\nabla^{2} B\right\|_{L^{2}}^{\frac{1}{2}}\|\nabla u\|_{L^{2}}\right) \\
& \leq \frac{1}{4}\left\|\nabla^{2} u\right\|_{L^{2}}+\frac{1}{4}\left\|\nabla^{2} B\right\|_{L^{2}}+C\left(\left\|B_{t}\right\|_{L^{2}}+\|\nabla B\|_{L^{2}}^{2}+\|\nabla u\|_{L^{2}}^{2}\right),
\end{aligned}
$$

where we have used (11) and (24). Similarly, we conclude from (12), (11), and (24) that

$$
\begin{aligned}
\left\|\nabla^{2} u\right\|_{L^{2}} & \leq C\left(\|\rho \dot{u}\|_{L^{2}}+\|B \cdot \nabla B\|_{L^{2}}\right) \\
& \leq C\left(\|\rho \dot{u}\|_{L^{2}}+\|B\|_{L^{\infty}}\|\nabla B\|_{L^{2}}\right) \\
& \leq C\left(\|\rho \dot{u}\|_{L^{2}}+\|B\|_{L^{2}}^{\frac{1}{2}}\left\|\nabla^{2} B\right\|_{L^{2}}^{\frac{1}{2}}\|\nabla B\|_{L^{2}}\right) \\
& \leq \frac{1}{4}\left\|\nabla^{2} B\right\|_{L^{2}}+C\left(\|\rho \dot{u}\|_{L^{2}}+\|\nabla B\|_{L^{2}}^{2}\right) .
\end{aligned}
$$

Hence, combining (25) and (26), we obtain

$$
\begin{aligned}
& \int_{0}^{T}\left(\left\|\nabla^{2} u\right\|_{L^{2}}^{2}+\left\|\nabla^{2} B\right\|_{L^{2}}^{2}\right) d \tau \\
& \quad \leq \sup _{s \leq \tau \leq t}\left(\|\nabla u\|_{L^{2}}^{2}+\|\nabla B\|_{L^{2}}^{2}\right) C_{0}+C \int_{s}^{t}\left(\|\sqrt{\rho} \dot{u}\|_{L^{2}}^{2}+\left\|B_{t}\right\|_{L^{2}}^{2}\right) d \tau .
\end{aligned}
$$

Thus, keeping the definition of $\Psi(t)$ in mind, we conclude from (27) that

$$
\begin{aligned}
& \|u\|_{L^{2}\left(s, t ; L^{\infty}\right)}^{2}+\|B\|_{L^{2}\left(s, t ; L^{\infty}\right)}^{2} \\
& \quad \leq C\left[1+\left(\|u\|_{L^{2}\left(s, t ; H^{1}\right)}^{2}+\|B\|_{L^{2}\left(s, t ; H^{1}\right)}^{2}\right)\right]\left[\ln \left(e+\|u\|_{L^{2}\left(s, t ; W^{1,4}\right)}\right)+\ln \left(e+\|B\|_{L^{2}\left(s, t ; W^{1,4}\right)}\right)\right] \\
& \quad \leq C\left[1+\left(\|u\|_{L^{2}\left(s, t ; H^{1}\right)}^{2}+\|B\|_{L^{2}\left(s, t ; H^{1}\right)}^{2}\right)\right]\left[\ln \left(e+\|u\|_{L^{2}\left(s, t ; H^{2}\right)}\right)+\ln \left(e+\|B\|_{L^{2}\left(s, t ; H^{2}\right)}\right)\right] \\
& \quad \leq C\left[1+\left(\|u\|_{L^{2}\left(s, t ; H^{1}\right)}^{2}+\|B\|_{L^{2}\left(s, t ; H^{1}\right)}^{2}\right)\right] \ln (C \Psi(t)) .
\end{aligned}
$$

Substituting the above estimate into (25), we conclude that

$$
\begin{aligned}
\Psi(t) & \leq C \Psi(s) \exp \left\{C_{1}\left[\|\nabla u\|_{L^{2}\left(s, t ; L^{2}\right)}^{2}+\|\nabla B\|_{L^{2}\left(s, t ; L^{2}\right)}^{2}\right] \ln \left(C_{2} \Psi(t)\right)\right\} \\
& \leq C \Psi(s)\left[C_{2} \Psi(t)\right]^{C_{1}\left(\|\nabla u\|_{L^{2}\left(s, t ; L^{2}\right)}^{2}+\|\nabla B\|_{L^{2}\left(s, t ; L^{2}\right)}^{2}\right)} .
\end{aligned}
$$

It follows from the basic energy estimate that one can choose the interval $[s, T]$ small enough, such that

$$
C_{1}\left(\|\nabla u\|_{L^{2}\left(s, t ; L^{2}\right)}^{2}+\|\nabla B\|_{L^{2}\left(s, t ; L^{2}\right)}^{2}\right) \leq \frac{1}{2}
$$


Substituting the above estimate into (25), we conclude that

$$
\Psi(T) \leq C \Psi(s) \Psi^{\frac{1}{2}}(T),
$$

which implies that

$$
\Psi(T) \leq C \Psi^{2}(s)
$$

from which we complete the proof of this lemma.

Remark 3.1 Due to (18) and the definition of the material derivative $\dot{f}$, we show that

$$
\int_{0}^{T}\left\|\sqrt{\rho} u_{t}\right\|_{L^{2}}^{2} d t \leq C
$$

by the following simple fact, i.e.:

$$
\begin{aligned}
\int_{0}^{T}\left\|\sqrt{\rho} u_{t}\right\|_{L^{2}}^{2} d t & \leq \int_{0}^{T}\|\sqrt{\rho} \dot{u}\|_{L^{2}}^{2} d t+\int_{0}^{T} \rho|u|^{2}|\nabla u|^{2} d x d t \\
& \leq \int_{0}^{T}\|\sqrt{\rho} \dot{u}\|_{L^{2}}^{2} d t+C \int_{0}^{T}\|u\|_{L^{4}}^{2}\|\nabla u\|_{L^{4}}^{2} d t \\
& \leq C+\int_{0}^{T}\|u\|_{L^{2}}\|\nabla u\|_{L^{2}}^{2}\left\|\nabla^{2} u\right\|_{L^{2}} d t \leq C,
\end{aligned}
$$

where we have used (9), (16), (17), and (18).

The following lemma is devoted to improving the time regularity of $u$ and $B$.

Lemma 3.4 For every $0<T<T^{*}$, we have

$$
\sup _{0 \leq t \leq T}\left(\left\|\sqrt{\rho} u_{t}\right\|_{L^{2}}^{2}+\left\|B_{t}\right\|_{L^{2}}^{2}\right)+\int_{0}^{T}\left(\left\|\nabla u_{t}\right\|_{L^{2}}^{2}+\left\|\nabla B_{t}\right\|_{L^{2}}^{2}\right) d t \leq C .
$$

Proof Differentiating (2) with respect to $t$, we obtain

$$
\rho u_{t t}+\rho u \cdot \nabla u_{t}+\nabla P_{t}-\Delta u_{t}=-\rho_{t} u_{t}-\rho_{t} u \cdot \nabla u-\rho u_{t} \cdot \nabla u+B_{t} \cdot \nabla B+B \cdot \nabla B_{t} .
$$

Multiplying the above equation by $u_{t}$, then integrating the resulting equation over $R^{2}$ on $x$, we deduce that

$$
\begin{aligned}
\frac{1}{2} & \frac{d}{d t}\left\|\sqrt{\rho} u_{t}\right\|_{L^{2}}^{2}+\left\|\nabla u_{t}\right\|_{L^{2}}^{2} \\
= & -\int \rho_{t}\left|u_{t}\right|^{2} d x-\int \rho_{t} u \cdot \nabla u \cdot u_{t} d x-\int \rho u_{t} \cdot \nabla u \cdot u_{t} d x \\
& +\int B_{t} \cdot \nabla B \cdot u_{t} d x+\int B \cdot \nabla B_{t} \cdot u_{t} d x \\
= & \sum_{i=1}^{5} I_{i} .
\end{aligned}
$$


Now, we estimate each term on the right-hand side of (31). First, due to (1), we have

$$
\begin{aligned}
I_{1} & =\int \operatorname{div}(\rho u)\left|u_{t}\right|^{2} d x=-2 \int \rho u \cdot u_{t} \cdot \nabla u_{t} \\
& \leq C\|\rho\|_{L^{\infty}}^{\frac{1}{2}}\left\|\sqrt{\rho} u_{t}\right\|_{L^{2}}\|u\|_{L^{\infty}}\left\|\nabla u_{t}\right\|_{L^{2}} \\
& \leq \frac{1}{8}\left\|\nabla u_{t}\right\|_{L^{2}}^{2}+C\|u\|_{L^{\infty}}^{2}\left\|\sqrt{\rho} u_{t}\right\|_{L^{2}}^{2} .
\end{aligned}
$$

Next, it follows from (1), (18), Hölder's inequality, and Young's inequality that

$$
\begin{aligned}
I_{2}= & \int \operatorname{div}(\rho u) u \cdot \nabla u \cdot u_{t} d x \\
\leq & \int \rho u|\nabla u|^{2}\left|u_{t}\right| d x+\int \rho|u|^{2}\left|\nabla^{2} u\right|\left|u_{t}\right| d x+\int \rho|u|^{2}\left|\nabla u \| \nabla u_{t}\right| d x \\
\leq & C\|\rho\|_{L^{\infty}}^{\frac{1}{2}}\|u\|_{L^{\infty}}\left\|\sqrt{\rho} u_{t}\right\|_{L^{2}}\|\nabla u\|_{L^{4}}^{2} \\
& +C\|u\|_{L^{\infty}}^{2}\left\|\sqrt{\rho} u_{t}\right\|_{L^{2}}\left\|\nabla^{2} u\right\|_{L^{2}}+C\|u\|_{L^{\infty}}^{2}\|\nabla u\|_{L^{2}}\left\|\nabla u_{t}\right\|_{L^{2}} \\
\leq & C\|u\|_{L^{\infty}}\left\|\sqrt{\rho} u_{t}\right\|_{L^{2}}\|\nabla u\|_{L^{2}}\left\|\nabla^{2} u\right\|_{L^{2}} \\
& +C\|\rho\|_{L^{\infty}}^{\frac{1}{2}}\|u\|_{L^{\infty}}^{2}\left\|\sqrt{\rho} u_{t}\right\|_{L^{2}}\left\|\nabla^{2} u\right\|_{L^{2}}+C\|u\|_{L^{\infty}}^{2}\|\nabla u\|_{L^{2}}\left\|\nabla u_{t}\right\|_{L^{2}} \\
\leq & \frac{1}{8}\left\|\nabla u_{t}\right\|_{L^{2}}^{2}+\left(1+\|u\|_{L^{\infty}}^{4}\right)\left\|\sqrt{\rho} u_{t}\right\|_{L^{2}}^{2}+C\left(1+\|\nabla u\|_{L^{2}}^{2}\right)\left\|\nabla^{2} u\right\|_{L^{2}}^{2} \\
\leq & \frac{1}{8}\left\|\nabla u_{t}\right\|_{L^{2}}^{2}+\left(1+\|u\|_{L^{2}}^{2}\left\|\nabla^{2} u\right\|_{L^{2}}^{2}\right)\left\|\sqrt{\rho} u_{t}\right\|_{L^{2}}^{2}+C\left(1+\|\nabla u\|_{L^{2}}^{2}\right)\left\|\nabla^{2} u\right\|_{L^{2}}^{2} .
\end{aligned}
$$

Then one obtains

$$
\begin{aligned}
I_{3} & \leq C\|\rho\|_{L^{\infty}}^{\frac{1}{2}}\left\|\sqrt{\rho} u_{t}\right\|_{L^{2}}\|\nabla u\|_{L^{4}}\left\|u_{t}\right\|_{L^{4}} \\
& \leq C\left\|\sqrt{\rho} u_{t}\right\|_{L^{2}}\|\nabla u\|_{L^{2}}^{\frac{1}{2}}\left\|\nabla^{2} u\right\|_{L^{2}}^{\frac{1}{2}}\left\|u_{t}\right\|_{L^{2}}^{\frac{1}{2}}\left\|\nabla u_{t}\right\|_{L^{2}}^{\frac{1}{2}} \\
& \leq C\left\|\sqrt{\rho} u_{t}\right\|_{L^{2}}\|\nabla u\|_{L^{2}}^{\frac{1}{2}}\left\|\nabla^{2} u\right\|_{L^{2}}^{\frac{1}{2}}\left(\left\|\sqrt{\rho} u_{t}\right\|_{L^{2}}^{\frac{1}{2}}+\left\|\nabla u_{t}\right\|_{L^{2}}^{\frac{1}{2}}\right)\left\|\nabla u_{t}\right\|_{L^{2}}^{\frac{1}{2}} \\
& =C\left\|\sqrt{\rho} u_{t}\right\|_{L^{2}}^{\frac{3}{2}}\|\nabla u\|_{L^{2}}^{\frac{1}{2}}\left\|\nabla^{2} u\right\|_{L^{2}}^{\frac{1}{2}}\left\|\nabla u_{t}\right\|_{L^{2}}^{\frac{1}{2}}+C\left\|\sqrt{\rho} u_{t}\right\|_{L^{2}}\|\nabla u\|_{L^{2}}^{\frac{1}{2}}\left\|\nabla^{2} u\right\|_{L^{2}}^{\frac{1}{2}}\left\|\nabla u_{t}\right\|_{L^{2}} \\
& \leq \frac{1}{8}\left\|\nabla u_{t}\right\|_{L^{2}}^{2}+C\left\|\sqrt{\rho} u_{t}\right\|_{L^{2}}^{2}\|\nabla u\|_{L^{2}}^{\frac{2}{3}}\left\|\nabla^{2} u\right\|_{L^{2}}^{\frac{2}{3}}+C\left\|\sqrt{\rho} u_{t}\right\|_{L^{2}}^{2}\|\nabla u\|_{L^{2}}\left\|\nabla^{2} u\right\|_{L^{2}} \\
& \leq \frac{1}{8}\left\|\nabla u_{t}\right\|_{L^{2}}^{2}+C\left(1+\left\|\nabla^{2} u\right\|_{L^{2}}^{2}\right)\left\|\sqrt{\rho} u_{t}\right\|_{L^{2}}^{2},
\end{aligned}
$$

where we have used (9), (11), and (24). Finally, as for $I_{4}$ and $I_{5}$, we see that

$$
\begin{aligned}
I_{4}+I_{5} & =\int B_{t} \cdot \nabla B \cdot u_{t}+\int B \cdot \nabla B_{t} \cdot u_{t} \\
& =-\int B_{t} \cdot \nabla u_{t} \cdot B-\int B \cdot \nabla u_{t} \cdot B_{t} \\
& \leq C\|B\|_{L^{\infty}}\left\|B_{t}\right\|_{L^{2}}\left\|\nabla u_{t}\right\|_{L^{2}} \\
& \leq \frac{1}{8}\left\|\nabla u_{t}\right\|_{L^{2}}^{2}+C\left(1+\left\|\nabla^{2} B\right\|_{L^{2}}^{2}\right)\left\|B_{t}\right\|_{L^{2}}^{2} .
\end{aligned}
$$


Hence, substituting all the above estimates into (31), we conclude that

$$
\begin{aligned}
\frac{d}{d t}\left\|\sqrt{\rho} u_{t}\right\|_{L^{2}}^{2}+\left\|\nabla u_{t}\right\|_{L^{2}}^{2} \leq & C\left(\left\|\nabla^{2} u\right\|_{L^{2}}^{2}+\left\|\nabla^{2} B\right\|_{L^{2}}^{2}\right)\left(\left\|\sqrt{\rho} u_{t}\right\|_{L^{2}}^{2}+\left\|B_{t}\right\|_{L^{2}}^{2}\right) \\
& +C\left(\left\|\sqrt{\rho} u_{t}\right\|_{L^{2}}^{2}+\left\|B_{t}\right\|_{L^{2}}^{2}\right) .
\end{aligned}
$$

From now on, we focus on the estimate for $B$. Differentiating equation (3) with respect to $t$, multiplying the resulting equation by $B_{t}$, and then integrating by parts over $\mathbb{R}^{2}$ on $x$, we finally obtain

$$
\begin{aligned}
& \frac{1}{2} \frac{d}{d t} \int\left|B_{t}\right|^{2} d x+\int\left|\nabla B_{t}\right|^{2} d x \\
& \quad=-\int u_{t} \cdot \nabla B \cdot B_{t} d x+\int\left(B_{t} \cdot \nabla u\right) \cdot B_{t} d x+\int(B \cdot \nabla) u_{t} \cdot B_{t} d x \\
& \quad=\sum_{i=1}^{3} J_{i} .
\end{aligned}
$$

We estimate each term on the right-hand side of (33). First, for $J_{1}$, ones deduce from (11) and (9) that

$$
\begin{aligned}
J_{1} & \leq C\left\|B_{t}\right\|_{L^{2}}\left\|u_{t}\right\|_{L^{4}}\|\nabla B\|_{L^{4}} \\
& \leq C\left\|B_{t}\right\|_{L^{2}}\left\|u_{t}\right\|_{L^{2}}^{\frac{1}{2}}\left\|\nabla u_{t}\right\|_{L^{2}}^{\frac{1}{2}}\|\nabla B\|_{L^{2}}^{\frac{1}{2}}\left\|\nabla^{2} B\right\|_{L^{2}}^{\frac{1}{2}} \\
& \leq C\left\|B_{t}\right\|_{L^{2}}\left(\left\|\sqrt{\rho} u_{t}\right\|_{L^{2}}^{\frac{1}{2}}+\left\|\nabla u_{t}\right\|_{L^{2}}^{\frac{1}{2}}\right)\left\|\nabla u_{t}\right\|_{L^{2}}^{\frac{1}{2}}\|\nabla B\|_{L^{2}}^{\frac{1}{2}}\left\|\nabla^{2} B\right\|_{L^{2}}^{\frac{1}{2}} \\
& \leq C\left\|B_{t}\right\|_{L^{2}}\left\|\sqrt{\rho} u_{t}\right\|_{L^{2}}^{\frac{1}{2}}\left\|\nabla u_{t}\right\|_{L^{2}}^{\frac{1}{2}}\|\nabla B\|_{L^{2}}^{\frac{1}{2}}\left\|\nabla^{2} B\right\|_{L^{2}}^{\frac{1}{2}}+C\left\|B_{t}\right\|_{L^{2}}\left\|\nabla u_{t}\right\|_{L^{2}}\|\nabla B\|_{L^{2}}^{\frac{1}{2}}\left\|\nabla^{2} B\right\|_{L^{2}}^{\frac{1}{2}} \\
& \leq C\left\|B_{t}\right\|_{L^{2}}\left\|\sqrt{\rho} u_{t}\right\|_{L^{2}}\|\nabla B\|_{L^{2}}^{\frac{1}{2}}\left\|\nabla^{2} B\right\|_{L^{2}}^{\frac{1}{2}}+C\left\|B_{t}\right\|_{L^{2}}\left\|\nabla u_{t}\right\|_{L^{2}}\|\nabla B\|_{L^{2}}^{\frac{1}{2}}\left\|\nabla^{2} B\right\|_{L^{2}}^{\frac{1}{2}} \\
& \leq \frac{1}{8}\left\|\nabla u_{t}\right\|_{L^{2}}^{2}+C\left(1+\left\|\nabla^{2} B\right\|_{L^{2}}^{2}\right)\left\|B_{t}\right\|_{L^{2}}^{2}+C\left\|\sqrt{\rho} u_{t}\right\|_{L^{2}}^{2} .
\end{aligned}
$$

Similarly, for $J_{2}$, we show that

$$
\begin{aligned}
J_{2} & \leq C\|\nabla u\|_{L^{2}}\left\|B_{t}\right\|_{L^{4}}^{2} \leq C\|\nabla u\|_{L^{2}}\left\|B_{t}\right\|_{L^{2}}\left\|\nabla B_{t}\right\|_{L^{2}} \\
& \leq \frac{1}{4}\left\|\nabla B_{t}\right\|_{L^{2}}^{2}+C\|\nabla u\|_{L^{2}}^{2}\left\|B_{t}\right\|_{L^{2}}^{2} .
\end{aligned}
$$

For $J_{3}$, one deduces

$$
J_{3} \leq C\|B\|_{L^{\infty}}\left\|\nabla u_{t}\right\|_{L^{2}}\left\|B_{t}\right\|_{L^{2}} \leq \frac{1}{8}\left\|\nabla u_{t}\right\|_{L^{2}}^{2}+C\left\|\nabla^{2} B\right\|_{L^{2}}^{2}\left\|B_{t}\right\|_{L^{2}}^{2}+C\left\|B_{t}\right\|_{L^{2}}^{2}
$$

Then, substituting the above estimates on $J_{1}, J_{2}, J_{3}$, one deduces

$$
\begin{aligned}
\frac{d}{d t} \int\left|B_{t}\right|^{2} d x+\int\left|\nabla B_{t}\right|^{2} d x \leq & \frac{1}{4}\left\|\nabla u_{t}\right\|_{L^{2}}^{2}+C\left(\|\nabla u\|_{L^{2}}^{2}+\left\|\nabla^{2} B\right\|_{L^{2}}^{2}\right)\left\|B_{t}\right\|_{L^{2}}^{2} \\
& +C\left(\left\|\sqrt{\rho} u_{t}\right\|_{L^{2}}^{2}+\left\|B_{t}\right\|_{L^{2}}^{2}\right) .
\end{aligned}
$$


Thus, combining (32) and (34), together with Gronwall's inequality, one easily completes the proof of (30). This completes the proof of Lemma 3.4.

Next, we will apply (12) and (13) to improve the higher regularity on the velocity $u$ and magnetic fields $B$, respectively.

Lemma 3.5 For every $0<T<T^{*}$, we have

$$
\sup _{0 \leq t \leq T}\left(\|u\|_{H^{2}}^{2}+\|B\|_{H^{2}}^{2}\right)+\int_{0}^{T}\left(\|\nabla u\|_{W^{1,4}}^{2}+\|\nabla B\|_{W^{1,4}}^{2}\right) d t \leq C .
$$

Proof Let us rewrite (2) in the following form:

$$
-\Delta u+\nabla P=-\rho u_{t}-\rho u \cdot \nabla u+B \cdot \nabla B .
$$

Then, using Lemma 2.4, we conclude that

$$
\begin{aligned}
\left\|\nabla^{2} u\right\|_{L^{2}} & \leq C\left(\left\|\rho u_{t}\right\|_{L^{2}}+\|\rho u \cdot \nabla u\|_{L^{2}}+\|B \cdot \nabla B\|_{L^{2}}\right) \\
& \leq C\left(\left\|\sqrt{\rho} u_{t}\right\|_{L^{2}}+\|u\|_{L^{4}}\|\nabla u\|_{L^{4}}+\|B\|_{L^{4}}\|\nabla B\|_{L^{4}}\right) \\
& \leq C\left(\left\|\sqrt{\rho} u_{t}\right\|_{L^{2}}+\left\|\nabla^{2} u\right\|_{L^{2}}^{\frac{1}{2}}+\left\|\nabla^{2} B\right\|_{L^{2}}^{\frac{1}{2}}\right) .
\end{aligned}
$$

Similarly, due to Lemma 2.5 , we obtain

$$
\begin{aligned}
\left\|\nabla^{2} B\right\|_{L^{2}} \leq & C\left(\left\|B_{t}\right\|_{L^{2}}+\|u \cdot \nabla B\|_{L^{2}}+\|B \cdot \nabla u\|_{L^{2}}\right) \\
\leq & C\left(\left\|B_{t}\right\|_{L^{2}}+\|u\|_{L^{4}}\|\nabla B\|_{L^{4}}+\|B\|_{L^{4}}\|\nabla u\|_{L^{4}}\right) \\
\leq & C\left(\left\|B_{t}\right\|_{L^{2}}+\|u\|_{L^{2}}^{\frac{1}{2}}\|\nabla u\|_{L^{2}}^{\frac{1}{2}}\|\nabla B\|_{L^{2}}^{\frac{1}{2}}\left\|\nabla^{2} B\right\|_{L^{2}}^{\frac{1}{2}}\right. \\
& \left.+\|B\|_{L^{2}}^{\frac{1}{2}}\|\nabla B\|_{L^{2}}^{\frac{1}{2}}\|\nabla u\|_{L^{2}}^{\frac{1}{2}}\left\|\nabla^{2} u\right\|_{L^{2}}^{\frac{1}{2}}\right) \\
\leq & C\left(\left\|B_{t}\right\|_{L^{2}}+\left\|\nabla^{2} B\right\|_{L^{2}}^{\frac{1}{2}}+\left\|\nabla^{2} u\right\|_{L^{2}}^{\frac{1}{2}}\right) .
\end{aligned}
$$

Thus, combining the above two inequalities and Young's inequality, we arrive at

$$
\sup _{0 \leq t \leq T}\left(\|u\|_{H^{2}}+\|B\|_{H^{2}}\right) \leq C .
$$

Then, by Lemmas 2.4 and 2.5 , we have

$$
\begin{aligned}
\int_{0}^{T}\left\|\nabla^{2} u\right\|_{L^{4}}^{2} d t \leq & C \int_{0}^{T}\left(\left\|\rho u_{t}\right\|_{L^{4}}^{2}+\|\rho u \cdot \nabla u\|_{L^{4}}^{2}+\|B \cdot \nabla B\|_{L^{4}}^{2}\right) d t \\
\leq & C \int_{0}^{T}\left(\left\|\sqrt{\rho} u_{t}\right\|_{L^{2}}^{2}+\left\|\nabla u_{t}\right\|_{L^{2}}^{2}+\|u\|_{L^{\infty}}^{2}\|\nabla u\|_{L^{4}}^{2}+\|B\|_{L^{\infty}}^{2}\|\nabla B\|_{L^{4}}^{2}\right) d t \\
\leq & C \int_{0}^{T}\left(\left\|\sqrt{\rho} u_{t}\right\|_{L^{2}}^{2}+\left\|\nabla u_{t}\right\|_{L^{2}}^{2}+\|u\|_{L^{2}}\left\|\nabla^{2} u\right\|_{L^{2}}\|\nabla u\|_{L^{2}}\left\|\nabla^{2} u\right\|_{L^{2}}\right. \\
& \left.+\|B\|_{L^{2}}\left\|\nabla^{2} B\right\|_{L^{2}}\|\nabla B\|_{L^{2}}\left\|\nabla^{2} B\right\|_{L^{2}}\right) d t \\
\leq & C+C \int_{0}^{T}\left\|\nabla u_{t}\right\|_{L^{2}}^{2} d t \leq C
\end{aligned}
$$


and

$$
\begin{aligned}
\int_{0}^{T}\left\|\nabla^{2} B\right\|_{L^{4}}^{2} d t \leq & C \int_{0}^{T}\left(\left\|B_{t}\right\|_{L^{4}}^{2}+\|u \cdot \nabla B\|_{L^{4}}^{2}+\|B \cdot \nabla u\|_{L^{4}}^{2}\right) d t \\
\leq & C \int_{0}^{T}\left(\left\|B_{t}\right\|_{L^{2}}^{2}+\left\|\nabla B_{t}\right\|_{L^{2}}^{2}+\|u\|_{L^{\infty}}^{2}\|\nabla B\|_{L^{4}}^{2}+\|B\|_{L^{\infty}}^{2}\|\nabla u\|_{L^{4}}^{2}\right) d t \\
\leq & C \int_{0}^{T}\left(\left\|B_{t}\right\|_{L^{2}}^{2}+\left\|\nabla B_{t}\right\|_{L^{2}}^{2}+\|u\|_{L^{2}}\left\|\nabla^{2} u\right\|_{L^{2}}\|\nabla B\|_{L^{2}}\left\|\nabla^{2} B\right\|_{L^{2}}\right. \\
& \left.+\|B\|_{L^{2}}\left\|\nabla^{2} B\right\|_{L^{2}}\|\nabla u\|_{L^{2}}\left\|\nabla^{2} u\right\|_{L^{2}}\right) d t \\
\leq & C+C \int_{0}^{T}\left\|\nabla B_{t}\right\|_{L^{2}}^{2} d t .
\end{aligned}
$$

Then, combining all the above estimates (36)-(40) together we show that (35). This completes the proof of Lemma 3.5.

Lemma 3.6 For every $0<T<T^{*}$, we have

$$
\sup _{0 \leq t \leq T}\left(\|\rho\|_{H^{2}}+\left\|\rho_{t}\right\|_{H^{1}}\right)+\int_{0}^{T}\left(\|u\|_{H^{3}}^{2}+\|B\|_{H^{3}}^{2}\right) d t \leq C .
$$

Proof Differentiating (1) with respect to $x_{i}(i=1,2)$, multiplying the resultant equation by $|\nabla \rho| \partial_{i} \rho$, then integrating the resulting equation by parts over $\mathbb{R}^{2}$ with respect to $x$, we finally deduce after summing them up that

$$
\frac{d}{d t}\|\nabla \rho\|_{L^{2}}^{2} \leq C\|\nabla u\|_{L^{\infty}}\|\nabla \rho\|_{L^{2}}^{2} \leq C\|\nabla u\|_{W^{1,4}}\|\nabla \rho\|_{L^{2}}^{2},
$$

which, combined with (35) and Gronwall's inequality, yields

$$
\|\nabla \rho\|_{L^{2}}^{2} \leq C\left\|\nabla \rho_{0}\right\|_{L^{2}}^{2} \exp \left\{C \int_{0}^{T}\|\nabla u\|_{W^{1,4}} d t\right\} \leq C .
$$

Similarly, we can also obtain from (1) that

$$
\begin{aligned}
\frac{d}{d t}\left\|\nabla^{2} \rho\right\|_{L^{2}}^{2} & \leq C\|\nabla u\|_{L^{\infty}}\left\|\nabla^{2} \rho\right\|_{L^{2}}^{2}+C\left\|\nabla^{2} u\right\|_{L^{4}}\|\nabla \rho\|_{L^{4}}\left\|\nabla^{2} \rho\right\|_{L^{2}} \\
& \leq C\|u\|_{W^{2,4}}\left(1+\left\|\nabla^{2} \rho\right\|_{L^{2}}^{2}\right),
\end{aligned}
$$

which combined with (42), together with (35) and Gronwall's inequality, yields

$$
\left\|\nabla^{2} \rho\right\|_{L^{2}}^{2} \leq C\left\|\nabla^{2} \rho_{0}\right\|_{L^{2}}^{2} \exp \left\{C \int_{0}^{T}\|u\|_{W^{2,4}} d t\right\} \leq C .
$$

It follows from (12) that

$$
\begin{aligned}
\|\nabla u\|_{H^{3}} \leq & C\left(\left\|\rho u_{t}\right\|_{H^{1}}+\|\rho u \cdot \nabla u\|_{H^{1}}+\|B \cdot \nabla B\|_{H^{1}}\right) \\
\leq & C\left(\left\|\rho u_{t}\right\|_{L^{2}}+\left\|\nabla \rho \cdot u_{t}\right\|_{L^{2}}+\left\|\rho \nabla u_{t}\right\|_{L^{2}}+\|\rho u \cdot \nabla u\|_{L^{2}}+\|\nabla \rho \cdot u \cdot \nabla u\|_{L^{2}}\right. \\
& \left.+\left\|\rho(\nabla u)^{2}\right\|_{L^{2}}+\left\|\rho u \cdot \nabla^{2} u\right\|_{L^{2}}+\|B \cdot \nabla B\|_{L^{2}}+\|\nabla B\|_{L^{4}}^{2}+\left\|B \cdot \nabla^{2} B\right\|_{L^{2}}\right)
\end{aligned}
$$




$$
\begin{aligned}
\leq & C\left(\|\rho\|_{L^{4}}^{\frac{1}{2}}\left\|\sqrt{\rho} u_{t}\right\|_{L^{2}}+\|\nabla \rho\|_{L^{4}}\left\|u_{t}\right\|_{L^{4}}\right. \\
& +\|\rho\|_{L^{\infty}}\left\|\nabla u_{t}\right\|_{L^{2}}+\|\rho\|_{L^{\infty}}\|u\|_{L^{\infty}}\|\nabla u\|_{L^{2}} \\
& +\|u\|_{L^{\infty}}\|\nabla \rho\|_{L^{4}}\|\nabla u\|_{L^{4}}+\|\rho\|_{L^{\infty}}\|\nabla u\|_{L^{4}}^{2}+\|\rho\|_{L^{\infty}}\|u\|_{L^{\infty}}\left\|\nabla^{2} u\right\|_{L^{2}} \\
& \left.+\|B\|_{L^{\infty}}\|\nabla B\|_{L^{2}}+\|\nabla B\|_{L^{4}}^{2}+\|B\|_{L^{\infty}}\left\|\nabla^{2} B\right\|_{L^{2}}\right) \\
\leq & C\left(1+\left\|\nabla u_{t}\right\|_{L^{2}}\right),
\end{aligned}
$$

which implies $\int_{0}^{T}\|u\|_{H^{3}} d t \leq C$. Similarly, we can obtain $\int_{0}^{T}\|B\|_{H^{3}} d t \leq C$. Thus, we obtain (41), and thus complete the proof of Lemma 3.6.

The proof of Theorem 1.1 is based on all the estimates that we deduced in Lemmas 3.13.6. From all the estimates obtained, we arrive at (15), and, finally, the proof of Theorem 1.1 is therefore completed.

\section{Competing interests}

The authors declare that they have no competing interests.

\section{Authors' contributions}

MS carried out the main work and drafted the manuscript. XQ participated in completing the proof of Lemma 3.5 JW participated in completing the proof of Lemma 3.6. All authors read and approved the final manuscript.

\section{Author details}

${ }^{1}$ School of Mathematical Sciences, Luoyang Normal University, Luoyang, 471022, P.R. China. ${ }^{2}$ College of Science, Zhongyuan University of Technology, Zhengzhou, 450007, P.R. China. ${ }^{3}$ School of Mathematics, Ocean University of China, Qingdao, 266100, P.R. China.

\section{Acknowledgements}

This work was supported by NSFC-Union Science Foundation of Henan (No. U1304103) and Natural Science Foundation of Henan Province (No. 122300410261).

\section{Received: 4 January 2014 Accepted: 31 March 2014 Published: 06 May 2014}

\section{References}

1. Cabannes, H: Theoretical Magnetofluiddynamics. Academic Press, New York (1970)

2. Kulikovskiy, AF, Lyubimov, GA: Magnetohydrodynamics. Addison-Wesley, Reading (1965)

3. Abidi, H, Paicu, M: Global existence for the magnetohydrodynamic system in critical spaces. Proc. R. Soc. Edinb., Sect. A 138, 447-476 (2008)

4. Chen, Q, Tan, Z, Wang, Y: Strong solutions to the incompressible magnetohydrodynamic equations. Math. Methods Appl. Sci. 34, 94-107 (2011)

5. Chen, G-Q, Wang, D: Global solution of nonlinear magnetohydrodynamics with large initial data. J. Differ. Equ. 182, 344-376 (2002)

6. Chen, G-Q, Wang, D: Existence and continuous dependence of large solutions for the magnetohydrodynamic equations. Z. Angew. Math. Phys. 54, 608-632 (2003)

7. Craig, W, Huang, X, Wang, Y: Global wellposedness for the 3D inhomogeneous incompressible Navier-Stokes equations. arXiv:1301.7155

8. Fan, J, Yu, W: Strong solution to the compressible MHD equations with vacuum. Nonlinear Anal., Real World Appl. 10, 392-409 (2009)

9. Hoff, D, Tsyganov, E: Uniqueness and continuous dependence of weak solutions in compressible magnetohydrodynamics. Z. Angew. Math. Phys. 56, 791-804 (2005)

10. Hu, X, Wang, D: Global solutions to the three-dimensional full compressible magnetohydrodynamic flows. Commun. Math. Phys. 283, 255-284 (2008)

11. Hu, X, Wang, D: Global existence and large-time behavior of solutions to the threedimensional equations of compressible magnetohydrodynamic flows. Arch. Ration. Mech. Anal. 197, 203-238 (2010)

12. Huang, X, Wang, Y: Global strong solution to the $2 D$ nonhomogeneous incompressible MHD system. J. Differ. Equ. 254, 511-527 (2013)

13. Jiang, S, Li, F: Convergence of the complete electromagnetic fluid system to the full compressible magnetohydrodynamic equations. arXiv:1309.3668

14. Kawashima, S: Smooth global solutions for two-dimensional equations of electromagnetofluid dynamics. Jpn. J. Appl. Math. 1, 207-222 (1984)

15. $\mathrm{Li}, \mathrm{H}, \mathrm{Xu}, \mathrm{X}$, Zhang, J: Global classical solutions to 3D compressible magnetohydrodynamic equations with large oscillations and vacuum. SIAM J. Math. Anal. 45, 1356-1387 (2013)

16. Wang, D: Large solutions to the initial-boundary value problem for planar magnetohydrodynamics. SIAM J. Appl. Math. 63, 1424-1441 (2003) 
17. Umeda, T, Kawashima, S, Shizuta, Y: On the decay of solutions to the linearized equations of electromagnetofluid dynamics. Jpn. J. Appl. Math. 1, 435-457 (1984)

18. Tsyganov, E, Hoff, D: Systems of partial differential equations of mixed hyperbolic-parabolic type. J. Differ. Equ. 204, 163-201 (2004)

19. Zhang, J: The inviscid and non-resistive limit in the Cauchy problem for 3-D nonhomogeneous incompressible magneto-hydrodynamics. Acta Math. Sci. 31, 882-896 (2011)

20. Zhang, J, Zhao, J: Some decay estimates of solutions for the 3-D compressible isentropic magnetohydrodynamics. Commun. Math. Sci. 8, 835-850 (2010)

21. Brezis, H, Wainger, S: A note on the limiting cases of Sobolev embedding and convolution inequalities. Commun. Partial Differ. Equ. 5, 773-789 (1980)

22. Adams, RA: Sobolev Space. Academic Press, New York (1975)

23. Galdi, GP: An Introduction to the Mathematical Theory of Navier-Stokes Equations. Volume I: Linearized Steady Problems. Springer Tracts in Natural Philosophy, vol. 38. Springer, Berlin (1994)

24. Ladyzhenskaya, OA: The Mathematical Theory of Viscous Incompressible Fluids, 2nd edn. Gordon \& Breach, New York (1969)

10.1186/1687-2770-2014-94

Cite this article as: Su et al.: Global existence of 2D nonhomogeneous incompressible magnetohydrodynamics with vacuum. Boundary Value Problems 2014, 2014:94

Submit your manuscript to a SpringerOpen ${ }^{\circ}$ journal and benefit from:

- Convenient online submission

- Rigorous peer review

- Immediate publication on acceptance

Open access: articles freely available online

- High visibility within the field

- Retaining the copyright to your article

Submit your next manuscript at $>$ springeropen.com 